$07,11,16,17$

\title{
Особенности зависимости рамановских спектров кластерных структур трехмерно-полимеризованного фуллерита от давления
}

\author{
(С) Ф.С. Хоробрых ${ }^{1,2}$, В.Д. Чуркин ${ }^{1,2}$, М.Ю. Попов ${ }^{1,2}$ \\ ${ }^{1}$ Московский фризико-технический институт (национальный исследовательский университет), \\ Долгопрудный, Россия \\ ${ }^{2}$ Технологический институт сверхтвердых и новых углеродных материалов, \\ Троицк, Россия \\ E-mail: fedor.khorobrykh@rambler.ru
}

Поступила в Редакцию 20 сентября 2021 г.

В окончательной редакции 29 октября 2021 г.

Принята к публикации 29 октября 2021 г.

\begin{abstract}
Исследовано влияние высокого гидростатического давления на трехмерно-полимеризованный фуллерит $3 \mathrm{D} \mathrm{C}_{60}$. После формирования $3 \mathrm{D} \mathrm{C}_{60}$ в условиях гидростатического нагружения при давлении $25 \mathrm{GPa}$ не наблюдается дальнейших структурных изменений, по крайней мере, до $150 \mathrm{GPa}$. Экспериментально показано, что полученные образцы состоят из отличающихся кластеров, образованных $s p^{3}$-связями с разным набором силовых констант, значения которых варьируются в пределах $20 \%$ и превышают силовые константы алмаза в 1.3-1.5 раза. Обнаружено влияние энергетической экспозиции лазерного излучения на процесс 3D-полимеризации $\mathrm{C}_{60}$ под давлением. Увеличение экспозиции в 15 раз приводит к снижению модуля объемного сжатия 3D $\mathrm{C}_{60}$ с $610 \mathrm{GPa}$ до $504 \mathrm{GPa}$.
\end{abstract}

Ключевые слова: модуль объемного сжатия, ультратвердый фуллерит, высокие давления, рамановская спектроскопия.

DOI: $10.21883 /$ FTT.2022.02.51933.207

\section{1. Введение}

Недавно была предложена фазовая диаграмма углерода, включающая новую область нестабильности алмаза $[1,2]$. Экспериментально доказано, что в диапазоне давлений от 55 до $115 \mathrm{GPa}$ формирование алмаза прекращается, а уже сформировавшиеся алмазы переходят в фуллереноподобные структуры, образованные под давлением $s p^{3}$-связями. Эти структуры могут включать фазы ультратвердого фуллерита, открытого более 25 лет назад [3]. Как показано в многочисленных экспериментальных исследованиях, ультратвердые состояния, получаемые из фуллерена $\mathrm{C}_{60}$, имеют значения твердости и модуля объемного сжатия в достаточно широких диапазонах $150-300 \mathrm{GPa}$ и $600-1000 \mathrm{GPa}$ соответственно в зависимости от условий воздействия на исходный $\mathrm{C}_{60}$ : от тензора напряжений, величины пластической деформации и температуры [3]. При этом повышение температуры до температуры Дебая алмаза в области стабильности алмаза 5-55 GPa приводит к частичной или полной трансформации ультратвердого фуллерита в алмаз в зависимости от времени воздействия высокой температуры [4,5].

Структурные исследования ультратвердого фуллерита затруднены, поскольку полученные образцы не имеют трансляционной симметрии на размерах больше $\sim 2 \mathrm{~nm}$ (по-видимому, это обусловлено тем, что $\mathrm{C}_{60}$ не имеет осей вращения четвертого порядка: только пятого, третьего и второго). Экспериментально показано, что ультратвердые состояния формируются в процессе 3D-полимеризации $\mathrm{C}_{60}$. При комнатной температуре это явление напрямую демонстрирует недавно обнаруженный эффект каталитической полимеризации и деполимеризации фуллерена. Как правило, для 3D $\mathrm{C}_{60}$ фаз наблюдаются искаженные ОЦК- и ГЦК-структуры, содержащие 70\% $s p^{3}$-связей [3].

Компьютерное моделирование показывает, что материалы, образованные углеродными нанокластерами, сформировавшиеся в результате 3D-полимеризации $\mathrm{C}_{60}$, могут иметь модуль объемного сжатия выше $1000 \mathrm{GPa}[6]$. Дальнейшие экспериментальные и теоретические исследования позволили установить, что высокие значения упругих модулей обусловлены возрастанием силовых констант по мере уменьшения размера углеродного нанокластера [7-9].

В связи с механическими и структурными особенностями ультратвердого фуллерита, возникает закономерный вопрос: какие структурные изменения могут происходить с ультратвердым фуллеритом как при давлениях 55-115 GPa, где стабильными являются фуллереноподобные фазы, так и при давлениях выше $115 \mathrm{GPa}$, когда алмаз снова стабилен. В настоящей работе мы экспериментально исследовали при давлениях до $150 \mathrm{GPa}$ упругие свойства нанокластеров, образующих ультратвердый $3 \mathrm{D} \mathrm{C}_{60}$, который формируется при комнатной температуре. 


\section{2. Эксперимент}

В работе использовался фуллерен $\mathrm{C}_{60}$ чистоты 99.99\%. Исследования при высоких давлениях проводились в камере с алмазными наковальнями (КАН) с использованием вольфрамовых гаскет. $\mathrm{C}_{60}$ нагружался в окружении $\mathrm{NaCl}$, который применяется в качестве среды, передающей давление. В экспериментах при давлениях до $150 \mathrm{GPa}$ использовались наковальни с фаской, размер рабочей площадки составлял $50 \mu \mathrm{m}$ (диаметр отверстия в гаскете $25 \mu \mathrm{m}$ ). В экспериментах до $80 \mathrm{GPa}$ использовались наковальни без фасок, размер рабочей площадки составлял $200 \mu \mathrm{m}$ (диаметр отверстия в гаскете - $70 \mu \mathrm{m})$. Рамановские спектры регистрировались на приборе Renishaw inVia. Длины волн возбуждающего излучения составляли 405 и $532 \mathrm{~nm}$. Лазер фокусировался в пятно на образце $1-2 \mu \mathrm{m}$. Давление определялось методом пьезоспектроскопии по Рамановским спектрам напряженной вершины алмазной наковальни [10].

\section{3. Результаты и обсуждение}

\section{1. Трансформации $\mathrm{C}_{60}$ при давлениях до $150 \mathrm{GPa}$}

Хорошо известно, что в условиях гидростатического нагружения при давлениях выше $25 \mathrm{GPa} \mathrm{C}_{60}$ переходит в ультратвердый $3 \mathrm{D} \mathrm{C}_{60}$ (при воздействии сдвиговых деформаций давление перехода уменьшается до $18 \mathrm{GPa}$ ) [5]. Этот эффект виден на рамановских спектрах, представленных на рис. 1. Спектр 3D $\mathrm{C}_{60}$ [11] в нашей работе наблюдается при давлении $25 \mathrm{GPa}$. Характерный спектр 3D $\mathrm{C}_{60}[11,12]$ состоит из двух широких полос около $1560 \mathrm{~cm}^{-1}$ и $600 \mathrm{~cm}^{-1}$ [11] (рис. 2). Регистрация спектров производилась при минимальной мощности лазера $1 \mathrm{~mW}$, позволяющей регистрировать спектры. Время накопления составляло $10 \mathrm{~min}$, длина волны возбуждающего излучения $532 \mathrm{~nm}$.

При увеличении давления выше $80 \mathrm{GPa}$ интенсивность высокочастотной моды $1560 \mathrm{~cm}^{-1}$ начинает уменьшаться и при давлениях выше $101 \mathrm{GPa}$ эта линия, как и низкочастотная мода около $600 \mathrm{~cm}^{-1}$, полностью исчезает (рис. 1). У нас пока нет оснований связывать исчезновение мод с фазовым переходом: давление $\sim 100 \mathrm{GPa}$ относится к области стабильности фуллереноподобных структур, и образование алмаза можно было бы ожидать при давлениях выше $115 \mathrm{GPa}[1,2]$. Однако и образование алмаза в данном эксперименте при давлениях 115-150 GPa не наблюдалось: при снятии давления рамановский спектр 3D $\mathrm{C}_{60}$ восстанавливается. На основании спектров (рис. 2) можно заключить, что нет отличий между образцами $3 \mathrm{D} \mathrm{C}_{60}$, полученными при сдвиге под давлением $20 \mathrm{GPa}[11]$ и при гидростатическом нагружении до $150 \mathrm{GPa}$.

Из зависимости рамановской частоты от давления (рис. 3) можно определить модуль объемного сжатия $B_{0}$

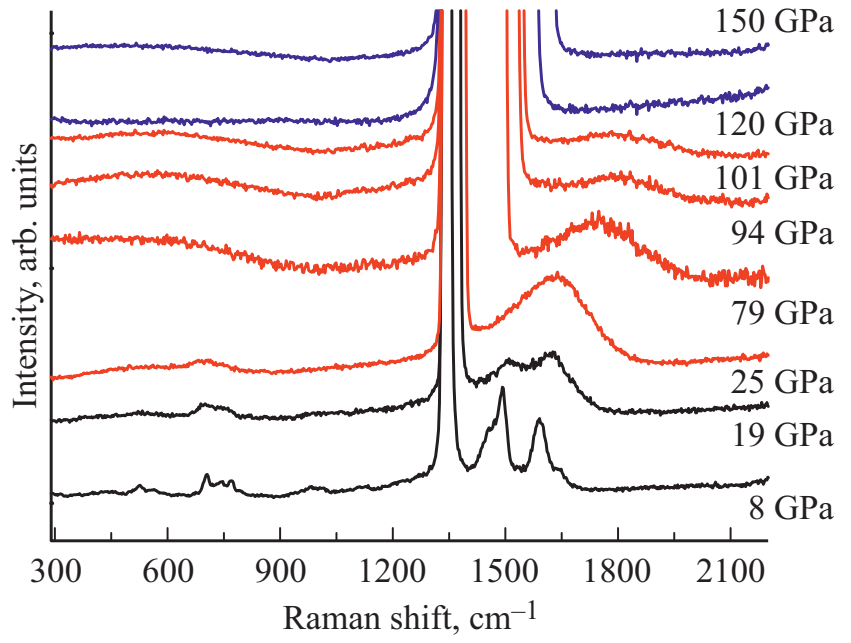

Рис. 1. Трансформация рамановских спектров фуллерена $\mathrm{C}_{60}$ с ростом давления.

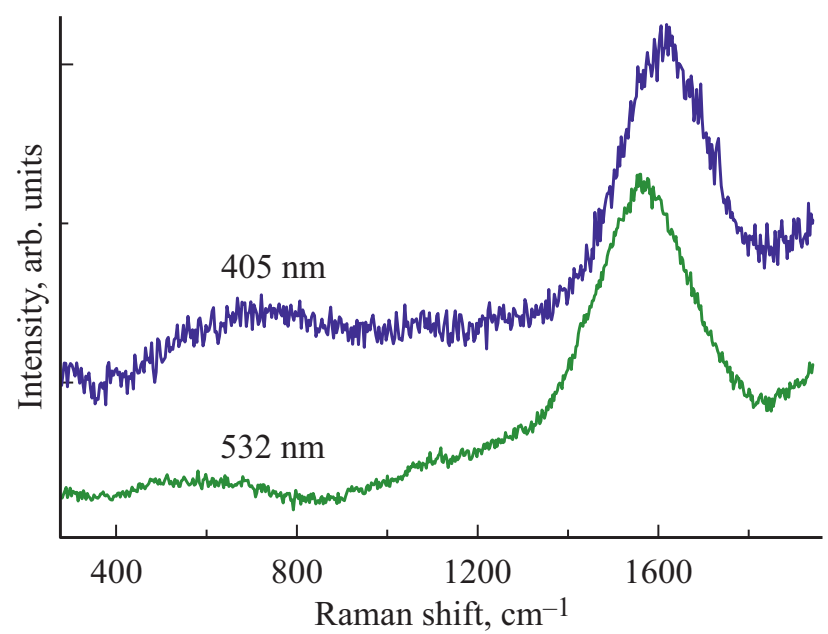

Рис. 2. Рамановские спектры образца $3 \mathrm{D} \mathrm{C}_{60}$. Спектры, полученные до снятия давления, снимались при мощности лазера $1 \mathrm{~mW}$, время накопления $10 \mathrm{~min}$, длины волн возбуждающего излучения 405 и $532 \mathrm{~nm}$.

материала по известному соотношению между коэффициентом Грюнайзена $\gamma_{i}$ и частотой $\omega_{i}$ фонона [13]:

$$
\gamma_{i}=-\frac{\partial \ln \omega_{i}}{\partial \ln V}=B_{0} \frac{\partial \ln \omega_{i}}{\partial P} .
$$

Для материалов, образованных ковалентно связанными атомами, $\gamma \approx 1$ [13] (в частности, для алмаза 0.96 и графита (вдоль графенового слоя) 1.1 [11]).

На рис. 3 нанесены точки, соответствующие 3D $\mathrm{C}_{60}$. Спектры снимались при мощности лазера $1 \mathrm{~mW}$, время накопления $10 \mathrm{~min}$, длины волн возбуждающего излучения $405 \mathrm{~nm}$ (треугольники) и $532 \mathrm{~nm}$ (кружки). Представлены данные, начиная с формирования фазы при $25 \mathrm{GPa}$ и заканчивая давлением $101 \mathrm{GPa}$, когда спектр 3D C 60 еще наблюдался при возбуждении $532 \mathrm{~nm}$, a также данные полученные при снятии давления. 


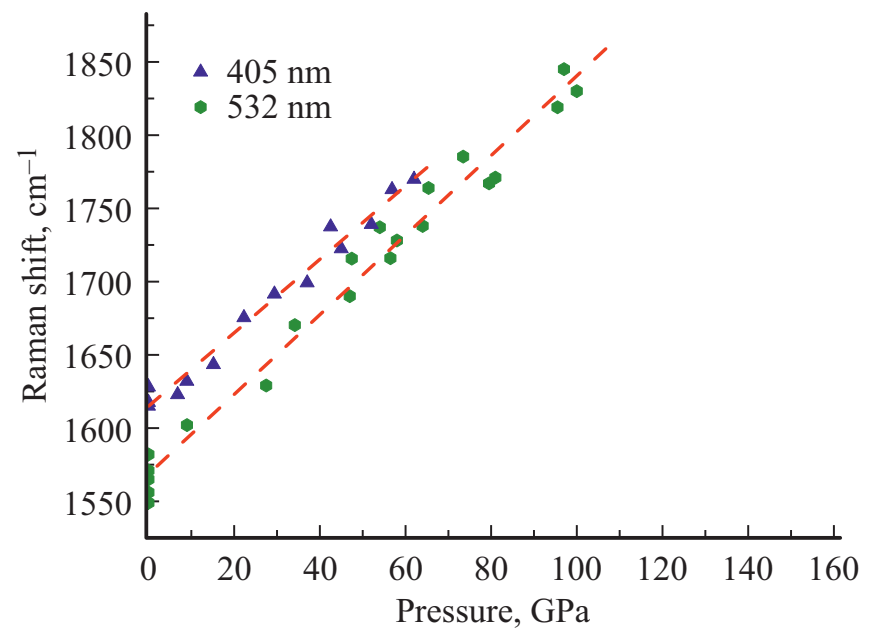

Рис. 3. Зависимость рамановской частоты $3 \mathrm{D} \mathrm{C}_{60}$ от давления. Спектры снимались при мощности лазера $1 \mathrm{~mW}$, время накопления $10 \mathrm{~min}$, длины волн возбуждающего излучения $405 \mathrm{~nm}$ (треугольники) и $532 \mathrm{~nm}$ (кружки). Представлены данные, начиная с формирования фазы при $25 \mathrm{GPa}$ и заканчивая давлением $101 \mathrm{GPa}$, когда спектр 3D $\mathrm{C}_{60}$ еще наблюдался.

Штриховой линией обозначена линейная интерполяция данных по методу наименьших квадратов. По наклону прямой на рис. 3 и из соотношения (1) при $\gamma=1$ получаем модуль объемного сжатия $B_{0}=577 \pm 19 \mathrm{GPa}$ для длины волны возбуждающего излучения $532 \mathrm{~nm}$, что близко к величине, полученной в работе [11] $(585 \mathrm{GPa})$. При этом в виду негидростатических условий нагружения в работе [11] была использована более сложная процедура оценки модуля объемного сжатия, включающая анализ тензоров напряжений образца и напряженной вершины алмазной наковальни.

Таким образом, в условиях гидростатического нагружения (при комнатной температуре) ультратвердый фуллерит сохраняется, по крайней мере, до $150 \mathrm{GPa}$. Формирование $3 \mathrm{D} \mathrm{C}_{60}$ происходит в первой области стабильности алмаза $(5-55 \mathrm{GPa})$ на фазовой диаграмме углерода $[1,2]$. Структура ультратвердого фуллерита не претерпевает заметных изменений в области неустойчивости алмаза (стабильности фуллереноподобных структур, 55-115 GPa) и сохраняется во второй области устойчивости алмаза (больше $115 \mathrm{GPa}$ ), оставаясь также в области стабильности графита (меньше $5 \mathrm{GPa}$ ). При этом образец, полученный в условиях гидростатического нагружения при давлении $25 \mathrm{GPa}$, эквивалентен образцу, полученному при инициализации 3D-полимеризации приложением сдвиговых деформаций при $20 \mathrm{GPa}$.

\section{2. Зависимость рамановских мод $3 \mathrm{D} \mathbf{C}_{60}$ от длины волны возбуждающего излучения и модуль объемного сжатия}

Наблюдаемая в спектрах $3 \mathrm{D} \mathrm{C}_{60}$ мода в районе $1560 \mathrm{~cm}^{-1}$ относится к $s p^{3}$-связям, как эксперименталь- но показано и детально обсуждалось в работе [7]. При смене длины волны возбуждающего излучения с зеленой (когда сечение рассеяния связей $s p^{2}$ на два порядка превосходит $s p^{3}$ ) на ультрафиолетовую $257 \mathrm{~nm}$ (при которой сечения рассеяния $s p^{2}-$ и $s p^{3}$-связей равны) ее интенсивность практически не меняется, зато наблюдается интересный эффект „резонансного“ смещения с $1550-1560 \mathrm{~cm}^{-1}$ на $1610-1640 \mathrm{~cm}^{-1}$ при длине волны возбуждающего излучения $257 \mathrm{~nm}[7,12]$ и на $1490 \mathrm{~cm}^{-1}$ при длине волны возбуждающего излучения $632 \mathrm{~nm}$ [4]. Компьютерное моделирование показывает, что углеродные нанокластеры имеют разные длины $s p^{3}$-связей в зависимости от расположения атомов (в частности, различаются длины связей вблизи центра и у поверхности) и, соответственно, разные наборы силовых констант $k$ [6-9]. При этом [14]. Для сравнения, Рамановская частота алмаза $1333 \mathrm{~cm}^{-1}$ и ковалентные связи в ультратвердом фуллерите в $1.3-1.5$ раза жестче, чем в алмазе. Таким образом, наблюдаемый эффект „резонансного“ смещения моды $1560 \mathrm{~cm}^{-1}$ может быть обусловлен зависимостью рамановского сечения рассеяния от особенностей локальных связей в нанокластерах. Другими словами, рамановский спектр $s p^{3}$-нанокластеров характеризуется широкой линией от $\sim 1490 \mathrm{~cm}^{-1}$ до $\sim 1640 \mathrm{~cm}^{-1}$ в соответствии с набором (тензором) силовых констант, значения которых различаются в пределах 20\% $\left(\omega^{2} \propto k\right)$. Смена длины волны возбуждающего излучения приводит к относительному росту интенсивности соответствующих участков одной широкой линии спектра. В нашей работе наблюдается смещение с $1568 \mathrm{~cm}^{-1}\left(\lambda_{\mathrm{ex}}=532 \mathrm{~nm}\right)$ на $1620 \mathrm{~cm}^{-1}\left(\lambda_{\mathrm{ex}}=405 \mathrm{~nm}\right)$ (рис. 2).

В целом, для определения упругих модулей при наличии в материале связей с разными силовыми константами, следует учитывать их суммарный вклад [14] или воспользоваться процедурой усреднения силовых констант в нанокластерах, предложенной в работе [9]. Для этого необходимо проводить анализ изменения не только частот, но и интенсивностей рамановских спектров при смене длины волны возбуждающего излучения, а также потребуются дополнительные данные о сечениях рассеяния в зависимости от величин силовых констант (или длин связей [9]).

Оценка модуля объемного сжатия по формуле (1) уже подразумевает усреднение по вкладам отдельных связей (со своими силовыми константами) в значение модуля. Если при этом значение модуля (из (1)) не будет зависеть от длины волны возбуждающего излучения, то можно говорить о точном значении полученного модуля объемного сжатия. В нашем случае картина более сложная. Модуль объемного сжатия, рассчитанный по формуле (1) (рис. 3), при длине волны возбуждающего излучения $\lambda_{\mathrm{ex}}=405 \mathrm{~nm} B_{0(405)}=644 \pm 27 \mathrm{GPa}$ и модуль при длине волны возбуждающего излучения $\lambda_{\mathrm{ex}}=532 \mathrm{~nm}$ $B_{0(532)}=577 \pm 19 \mathrm{GPa}$. Таким образом, модуль объемного сжатия, рассчитанный из соотношения рамановской 
частоты и давления, зависит от длины волны возбуждающего излучения. Разница в полученных значениях модулей превышает ошибку эксперимента. Наблюдаемый эффект можно объяснить тем, что структура образца состоит из двух типов кластеров, образованных связями с разным набором силовых констант. В соответствии с предложенной в работе [9] процедурой усреднения силовых констант, модуль объемного сжатия образца можно оценить как $B_{0}=0.5\left(B_{0(405)}+B_{0(532)}\right)=610 \mathrm{GPa}$.

Появление разных типов кластеров обусловлено $3 \mathrm{D}$-полимеризацией $\mathrm{C}_{60}$. $\mathrm{B}$ работе [15] предложен вариант ОЦК-структуры, образованной молекулами $\mathrm{C}_{60}$, связанными по механизму $(6+6)$. Подобная 3D-полимеризация приводит к тому, что между $\mathrm{C}_{60}$ образуются многогранники меньшего размера, образованные фрагментами $\mathrm{C}_{60}$ и 4-х членными кольцами, которые соединяют соседние $\mathrm{C}_{60}$. На каждую ОЦК-ячейку такой структуры приходится 6 искаженных усеченных октаэдров (состоящих из 8 гексагонов и 6 квадратов, 24 атомов, диаметром около $4.5 \AA$ ) и шести 12-гранников (состоящих из 4 гексагонов, 4 пентагонов и 4 квадратов, диаметром около $4 \AA$ ) [15]. Угол ковалентной связи в подобных фуллереноподобных близок к тетраэдрическому расположению $s p^{3}$ [16-18]. Поэтому для описания аморфной $3 \mathrm{D} \mathrm{C}_{60}$-структуры можно воспользоваться моделью хаотической плотной упаковки жестких сфер [19,20]. В рамках этой модели пространство может быть заполнено полиэдрами Бернала, которые содержат атомные сферы на каждой из вершин. Половина пространства полостей аморфного материала приходится на октаэдрические и тетраэдрические полости в соотношении $1: 2.5$, а половина — на сложные полиэдры.

\section{3. Эффект фотоиндуцированной 3D-полимеризации $\mathrm{C}_{60}$}

После формирования ультратвердого фуллерита технически невозможно использовать методику инициализации фазового перехода приложением сдвиговых деформаций, поскольку при вращении наковален в сдвиговой камере с алмазными наковальнями образец проскальзывает по алмазным наковальням, вызывая пластическую деформацию алмаза, а не $3 \mathrm{D} \mathrm{C}_{60}[3,11,12]$. В работе [7] был обнаружен эффект фотоинициализации перехода алмаза в фуллереноподобные структуры при давлениях 55-115 GPa. Такой же метод был использован и в настоящей работе. Образец $\mathrm{C}_{60}$ нагружался в условиях гидростатики (в окружении $\mathrm{NaCl}$, как и в предыдущем опыте). При давлениях выше $25 \mathrm{GPa}$ регистрация спектров производилась при мощности лазера $15 \mathrm{~mW}$ в течение $10 \mathrm{~min}$, как и в работе [7].

На рис. 4 приведены зависимости рамановской частоты 3D $\mathrm{C}_{60}$ от давления при длинах волн возбуждающего излучения 405 и $532 \mathrm{~nm}$. Пунктирными линиями обозначены линейные интерполяции данных для 405 и $532 \mathrm{~nm}$ по методу наименьших квадратов. Энергетическая экспозиция и интенсивность излучения при

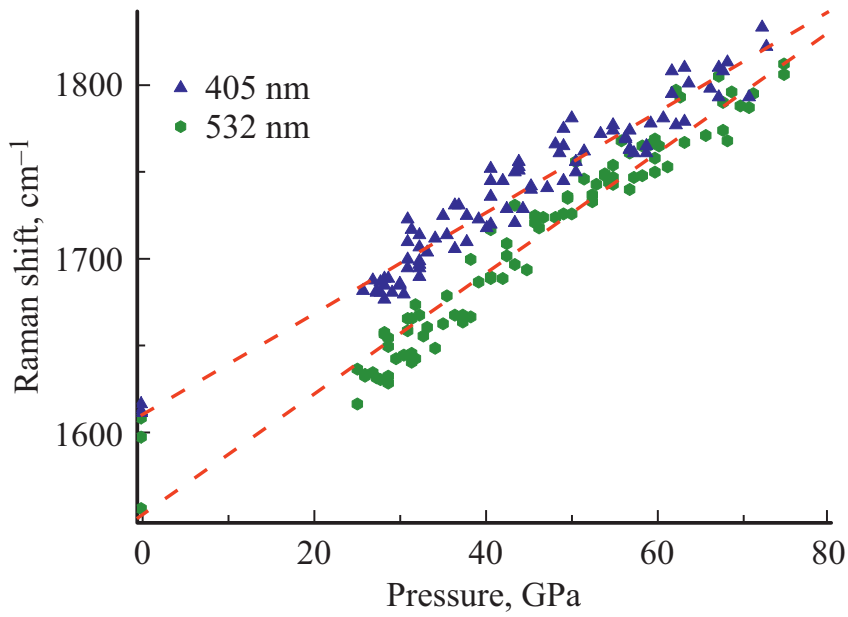

Рис. 4. Зависимость рамановской частоты $3 \mathrm{D} \mathrm{C}_{60}$ от давления. Спектры снимались при мощности лазера $15 \mathrm{~mW}$, время накопления $10 \mathrm{~min}$, длины волн возбуждающего излучения $405 \mathrm{~nm}$ (треугольники) и $532 \mathrm{~nm}$ (кружки).

накоплении спектров в этом опыте в 15 раз больше, чем в предыдущем. В условиях увеличенной экспозиции пик в районе $1560 \mathrm{~cm}^{-1}$ полностью исчезает из рамановского спектра под давлением $75 \mathrm{GPa}$. Для сравнения, в предыдущем опыте при меньшей экспозиции этот пик пропадал под давлением больше $101 \mathrm{GPa}$. Наблюдаемое исчезновение пика является обратимым. При снижении давления линия около $1560 \mathrm{~cm}^{-1}$ снова проявляется в спектрах.

Модуль объемного сжатия, рассчитанный по формуле (1) (рис. 4), при длине волны возбуждающего излучения $\lambda_{\mathrm{ex}}=405 \mathrm{~nm} B_{0(405)}=558 \pm 14 \mathrm{GPa}$ и модуль при длине волны возбуждающего излучения $\lambda_{\mathrm{ex}}=532 \mathrm{~nm}$ $B_{0(532)}=449 \pm 12 \mathrm{GPa}$. Как и в предыдущем случае, структура образца состоит из двух типов кластеров, образованных связями с разным набором силовых констант. Модуль объемного сжатия образца, полученного при повышенной энергетической экспозиции под давлением, $B_{0}=0.5\left(B_{0(405)}+B_{0(532)}\right)=504 \mathrm{GPa}$. Таким образом, увеличение энергетической экспозиции в 15 раз приводит к снижению модуля объемного сжатия с 610 до $504 \mathrm{GPa}$.

\section{4. Заключение}

Экспериментально показано, что 3D $\mathrm{C}_{60}$ образован $s p^{3}$-связями, значения силовых констант которых различаются в пределах 20\% и превышают силовые константы алмаза в $1.3-1.5$ раза.

Модуль объемного сжатия $3 \mathrm{D} \mathrm{C}_{60}$, рассчитанный из соотношения рамановской частоты и давления, зависит от длины волны возбуждающего излучения. Наблюдаемый эффект можно объяснить тем, что структура образца состоит, по крайней мере, из двух типов класте- 
ров, образованных связями с разным набором силовых констант.

После формирования 3D $\mathrm{C}_{60}$ в условиях гидростатического нагружения при давлении $25 \mathrm{GPa}$ не наблюдается дальнейших структурных изменений, по крайней мере, до $150 \mathrm{GPa}$.

Обнаружено влияние энергетической экспозиции лазерного излучения на $\mathrm{C}_{60}$ под давлением. Увеличение экспозиции в 15 раз приводит к снижению модуля объемного сжатия 3D $\mathrm{C}_{60}$ с 610 до $504 \mathrm{GPa}$.

\section{Финансирование работы}

Работа выполнена в рамках госзадания ФГБНУ ТИСНУМ.

\section{Конфликт интересов}

Авторы заявляют, что у них нет конфликта интересов.

\section{Список литературы}

[1] V.D. Blank, V.D. Churkin, B.A. Kulnitskiy, I.A. Perezhogin, A.N. Kirichenko, V.N. Denisov, S.V. Erohin, P.B. Sorokin, M.Yu. Popov. Nanotechnology 29, 115603 (2018).

[2] M.Yu. Popov, V.D. Churkin, B.A. Kulnitskiy, A.N. Kirichenko, K.M. Bulatov, A.A. Bykov, P.V. Zinin, V.D. Blank. Nanotechnology 31, 315602 (2020).

[3] M.Yu. Popov, B.A. Kulnitskiy, V.D. Blank. Comprehensive Hard Materials. Elsevier, Netherlands (2014). 515 p.

[4] V.D. Blank, S.G. Buga, G.A. Dubitsky, N.R. Serebryanaya, S.N. Sulyanov, M.Yu. Popov, V.N. Denisov, A.N. Ivlev, B.N. Mavrin. Phys. Lett. A 220, 149 (1996).

[5] B. Sundqvist. Phys. Rep. 909, 1 (2021).

[6] Y.A. Kvashnina, A.G. Kvashnin, M.Yu. Popov, B.A. Kulnitskiy, I.A. Perezhogin, E.V. Tyukalova, L.A. Chernozatonskii, P.B. Sorokin, V.D. Blank. J. Phys. Chem. Lett. 6, 2147 (2015).

[7] M. Popov, V. Churkin, A. Kirichenko, V. Denisov, D. Ovsyannikov, B. Kulnitskiy, I. Perezhogin, V. Aksenenkov, V. Blank. Nanoscale Res. Lett. 12, 561 (2017).

[8] M. Popov, V. Churkin, D. Ovsyannikov, A. Khabibrakhmanov, A. Kirichenko, E. Skryleva, Y. Parkhomenko, M. Kuznetsov, S. Nosukhin, P. Sorokin, S. Terentiev, V. Blank. Diamond Rel. Mater. 96, 52 (2019).

[9] A. Khabibrakhmanov, P. Sorokin. Carbon 160, 228 (2020).

[10] M. Popov. J. Appl. Phys. 95, 5509 (2004).

[11] M. Popov, M. Kyotani, Y. Koga. Diamond Rel. Mater. 12, 833 (2003).

[12] M. Popov, V. Mordkovich, S. Perfilov, A. Kirichenko, B. Kulnitskiy, I. Perezhogin, V. Blank. Carbon 76, 250 (2014).

[13] B.A. Weinstein, R. Zallen. Light Scattering in Solids. Springer, Berlin (1984). V. IV. 543 p.

[14] A.A. Maradudin, E.W. Montroll, G.H. Weiss. Solid State Physics. Academic Press, N.Y., London (1963). 319 p.

[15] M. O’Keeffe. Nature 352, 674 (1991).

[16] S. Park, D. Srivastava, K. Cho. J. Nanosci. Nanotech. 1, 1 (2001).

[17] C. Piskoti, J. Yarger, A. Zettl. Nature 393, 771 (1998).
[18] Z. Iqbal, Y. Zhang, H. Grebel, S. Vijayalakshmi, A. Lahamer, G. Benedek, M. Bernasconi, J. Cariboni, I. Spagnolatti, R. Sharma, F.J. Owens, M.E. Kozlov, K.V. Rao, M. Muhammed. Eur. Phys. J. B 31, 509 (2003).

[19] К. Судзуки, Х. Фудзимори, К. Хасимото. Металлургия, М. (1987). $328 \mathrm{c}$.

[20] А.М. Глезер, Б.В. Молотилов. Металлургия, М. (1992). $208 \mathrm{c}$.

Редактор Т.Н. Василевская 